\title{
Adequate Theory of Vertical Motion of a Mass-Elastic System
}

\author{
Zdzisław Pluta, Tadeusz Hryniewicz* \\ Koszalin University of Technology, Raclawicka 15-17, PL 75-620 Koszalin, Poland \\ *E-mail address: Tadeusz.Hryniewicz@tu.koszalin.pl
}

Dash with rules, we want to do something!

Thomas Edison

\begin{abstract}
The paper covers an adequate theory on vertical motion of the mass-elastic system. This system is an oscillator with the motion being a free harmonic motion. The first extended link of the consideration presents a critical analysis of the existent knowledge referred to this subject. Then the energetic states of the oscillator have been determined. Next the force characteristics of the free harmonic motion of the oscillator are presented. A general source equation of the path/way length and the resulting detailed forms, signalizing extensive possibilities to build up the characteristics, have been presented. Finally the connections between the characteristics of real and simulative motions were derived. It should be added that this first mentioned motion is the subject of adequate description presented here. The simulated motion is referred to the existent classical theory which has been subjected under a critical consideration.
\end{abstract}

Keywords: Theory of oscillation motion; Vertical mass-elastic system; Free harmonic oscillator motion; Potential field; Force characteristics; Source equation of path/way length; Time constant

\section{INTRODUCTION}

Determination of acceleration in science and technique development is always timely and fundamental task performed on the ground of creative activity. It has been always indicated on the need to develop this type of technical culture sphere. However, the most important factors are often omitted, which not only retard the development of science but even block or freeze it. They are just grounded rules (see the Thomas Edison aphorism), schemes, and paradigms which are the factors of pejorative character.

The development of science requires to break the rooted existent rules, laws, principles, theories and all other forms of description of the reality which are inadequate in character. It appears sometimes it is necessary to return to the source to get out again on the cognitive path.

There are lots of methodic needs for the activities. It is impossible to do all of these herewith to reveal their inadequacies, first of all due to the limited space for the description. Thus only one example is to be presented in this work. It is concerned with the vibrations, and particularly with the title problem, the theory of vertical motion of the mass-elastic system. 
The theory of vibration is based on a classical description with an erroneous fictional paradigm/theorem. It concerns the d'Alembert's rule which introduces a fictional inertia force to the system. Some exemplary sources of knowledge on material bodies [1-4] may illustrate the attachment to such a fictional-real interpretation of the reality. Other references, articles, e.g. [5-10] also prove of that. It is worth mentioning the work [5] which discusses the nonlinear vibrations as the open problem. The author [5] presents some basic directions of the studies of oscillation phenomenon, and in the summary he states that the viewpoint has changed. It is not about how to solve a set of non-linear differential equations but rather what model to use to describe the reality with a required accuracy. Is there more philosophy needed to approach this problem? The proper reasoning and understanding with the return to the source should adequately describe the studied reality.

There is no other way to reach the truth. Mathematical and complex descriptions of vibrations may veil the reality attempting only its proper interpretation. Therefore the mathematical tools should be used by a critical way to master the contents.

\section{EXISTENT STATE OF KNOWLEDGE}

The existent knowledge has been referred to the description of free vibrations of a vertical mass-elastic system. The system (Fig. 1) consists of a weight of mass $m$ fixed on the free end of a flat spring fixed one-sided way.

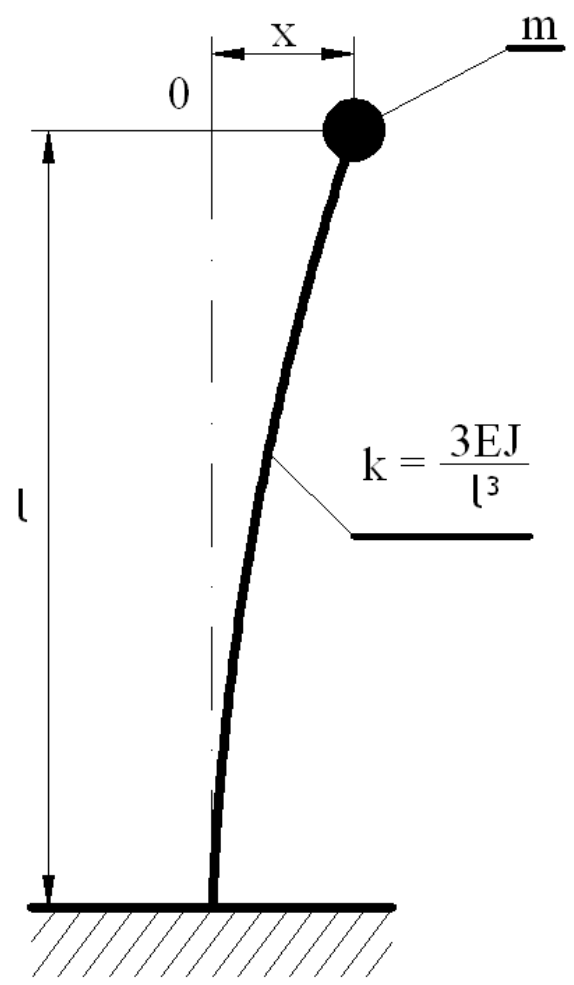

Fig. 1. Vertical mass-elastic system deflected from the equilibrium state.

The motion equation of this weight, in accordance to the commonly used d'Alemert's rule, has the following form

$$
m \ddot{x}+k x=0
$$


where: $\ddot{x}$ - fictional acceleration of the weight, $x$ - its deflection, $m$ - weight mass, $k-$ stiffness coefficient of elastic element, described here by the formula

$$
k=\frac{3 E J}{l^{3}}
$$

where $E$ means the longitudinal modulus of elasticity (Young's modulus) of the spring material, $J-$ is the so called axial moment of inertia of the spring cross-section, $l$ - the spring length.

Further progress, connected with the mathematical treatment of equation (1), is commonly known and rooted in numerous references. It is known that further analysis covers three particular cases of vibrations, differing by the so called initial/primary conditions. The primary conditions are assumed to be in connection with the beginning of measurement of a determined magnitude. They are known commonly that way which unfortunately is erroneous, concerning herewith the vibrating motion.

It is apparent also that at moderate deflections of this system from the position of equilibrium, this system performs a particular free motion, known as a simple harmonic motion (SHM). Taylor [1] introduces here the SHM and defines that free motion as described by functions of type sinus and cosine.

The analyses of references concerned with this problem reveal no basis in the essence of the free harmonic motion. They focus on mathematical solutions only which lead to determined results but these results are inadequate in character, that is they do not conform with the studied reality. This reality is under veil then. The physical aspect has been reduced to a determined part of the reality. It is important that the whole description of the reality is adequate. Then it may be recognized as an adequate theory, being under no cognitive conflict.

The equation (1) describes de facto a fictional rotation motion the existence of which is not possible at all. The form of this equation indicates that it refers to the description of rotational motion projection on the direction of oscillation of a considered system. Thus the amplitude of harmonic motion corresponds with the radius of track of rotational motion of weight fixed on the free end of spring.

That fictional-real equilibrium of weight corresponds with the identity/equality of decrease of the inertia force $\Delta B$ and the increase of elastic force $\Delta S$ (Fig. 2), that is

$$
\Delta B=\Delta S
$$

That fictional-real equilibrium may be specified as follows:

$$
m \omega^{2} A=k A
$$

where the symbol $A$ means the radius of path of weight in its rotational motion, and $\omega$ is the angular velocity of the whole system.

That velocity is defined by a formula of the form as follows

$$
\omega=\frac{2 \pi}{T_{0}}
$$




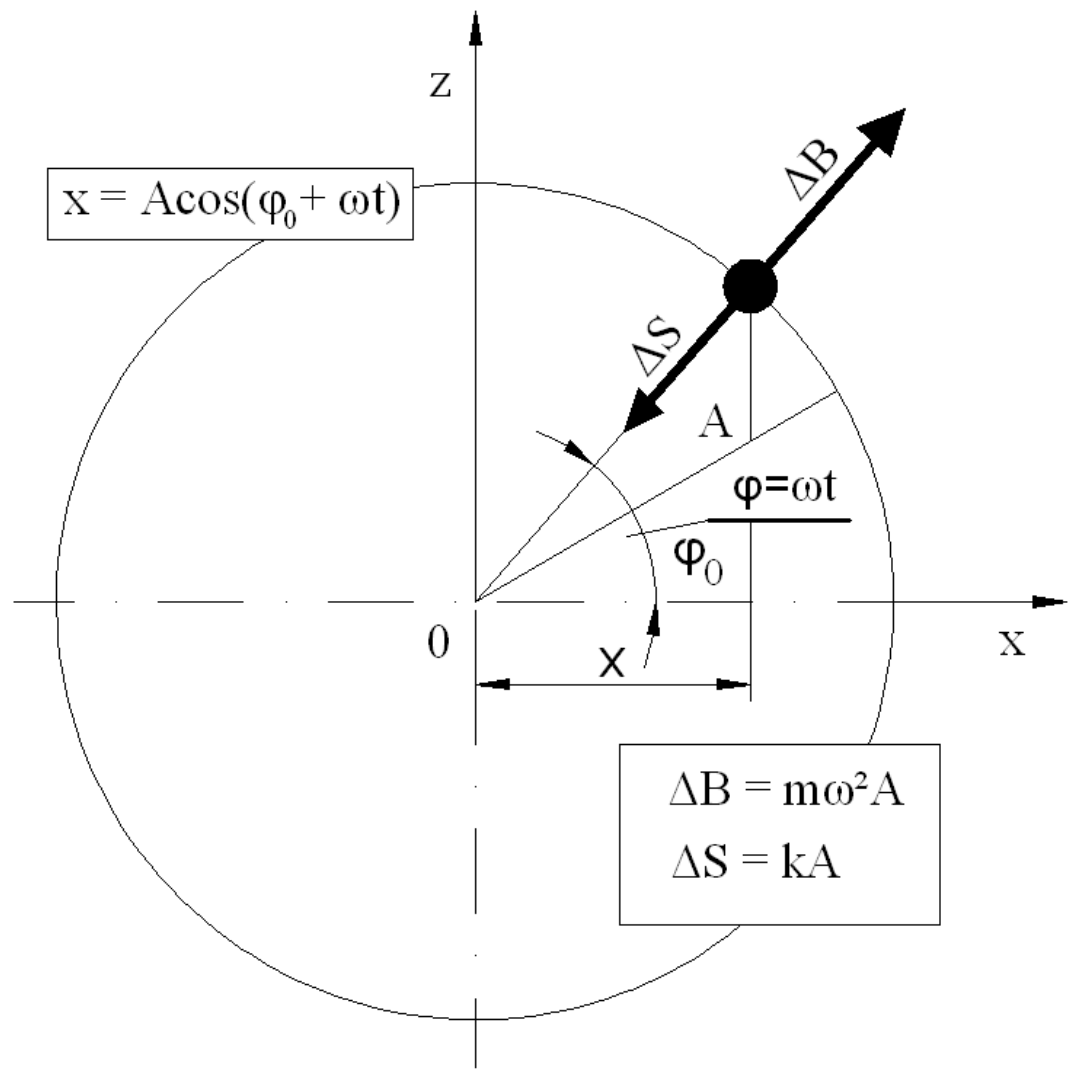

Fig. 2. Fictional-real equilibrium of weight in the rotational motion of the vertical mass-elastic system.

where $T_{0}$ is the period of rotational motion, that is the time of one complete cycle of weight on the path of the radius $A$.

Naturally, it results from equation (4) that

$$
\omega=\sqrt{\frac{k}{m}}
$$

which is commonly named as a circular or angular frequency, the pulsation of vibration or the frequency of free vibration.

That period of rotational motion $T_{0}$ is called the period of free vibration. The formula on this apparent magnitude results from the formula (5), after substituting to it the relationship (6). Here is a final, properly constructed form

$$
T_{0}=2 \pi \sqrt{\frac{m}{k}}
$$

from which it results that the period $T_{0}$ does not depend on the initial deflection but only on the mass $m$ and constant $k$.

In the analysis, the motion of weight projection corresponding with the equilibrium $\Delta B_{x}=\Delta S_{x}$ is a simplification. The record of a coordinate $x$ of the path of its motion is needed. That coordinate is determined by the following equation: 


$$
x=A \cos \left(\varphi_{0}+\varphi\right)=A \cos \left(\varphi_{0}+\omega t\right)
$$

where: $\varphi_{0}$ - initial phase, $\varphi$ - phase angle, $t$ - time of the body motion, with, as it is apparent, $\varphi=\omega t$.

Now, without any problem, further detailed variants of the description may be introduced into this fictional-real space. Before that, however, the relationship (8) should be developed, in accordance to the knowledge on the trigonometric function of sum of two angles. Here it is

$$
x=A \cos \varphi_{0} \cos \omega t-A \sin \varphi_{0} \sin \omega t
$$

or

$$
x=B_{1} \cos \omega t-B_{2} \sin \omega t
$$

because

$$
B_{1}=A \cos \varphi_{0}, B_{2}=A \sin \varphi_{0}
$$

Furthermore the derivative of this function, being the dependence of the coordinate $x$ on time $t$ may be derived. It has been assumed that the derivative is the so called the weight velocity, so

$$
\dot{x}=\frac{d x}{d t}=-B_{1} \omega \sin \omega t-B_{2} \omega \cos \omega t
$$

It is time now to analyze these three particular variants of the fictional-real oscillation motion. Their graphic interpretation (Fig. 3) will allow to observe the course of the procedure/progress connected with that cognitive question.

The first variant of commonly understood initial conditions assumes that at the initial moment $t=0$ it is $x=A, \dot{x}=0$. That simply means the initial measurement point is placed in the site where the coordinate of projection of weight position is maximum $(x=A)$, whereas the projection of velocity in this point assumes the value of zero $(\dot{x}=0)$.

By substituting these initial motion parameters to equations (10), and (12), one obtains

$$
B_{1}=A, B_{2}=0
$$

which further, after complying it with the equation (10), gives

$$
x=A \cos \omega t
$$

Let us now, at the moment $t=0$ it is $x=0, \dot{x}=v$. In accordance to the existent knowledge that means in the central position the initial velocity $v$ is promoted to the weight, e.g. by a hit. Thus we obtain

$$
B_{1}=0, B_{2}=-\frac{v}{\omega}=-A
$$



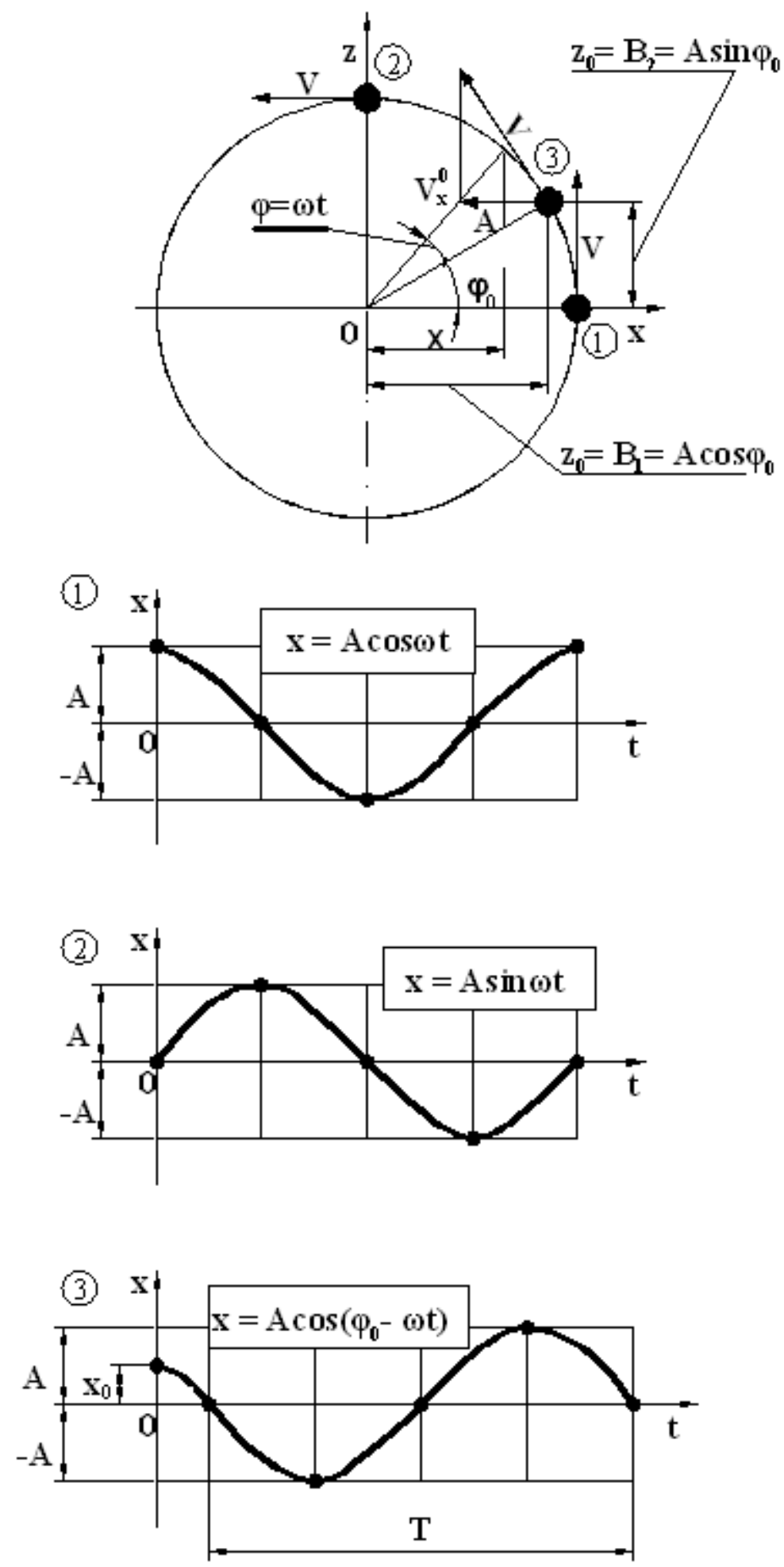

Fig. 3. Graphic interpretation of particular variants of fictional-real oscillation motion.

The equation of weight motion takes the form

$$
x=\frac{v}{\omega} \sin \omega t=A \sin \omega t
$$


and after taking into account the formula (6) on the so called frequency of free vibration

$$
x=v \sqrt{\frac{m}{k}} \sin \omega t
$$

One should add that in this case the initial measurement conditions took place in the middle of vibrations of the weight projection, i.e. in the place which was reached by the weight after passing the angular way $\varphi=\pi / 2 \mathrm{rad}$. Hence here in the assumed system of coordinates $t=0$, and $\dot{x}=v$. According to the third, indirect version of initial conditions, at the moment of $t=0$ one obtains $x=x_{0}, \dot{x}=v_{x}^{0}=v \sin \varphi_{0}$. For them, after complying with the equations (10), and (12), one obtains

$$
B_{1}=x_{0}, B_{2}=-\frac{v_{x}^{0}}{\omega}
$$

and then, after substituting of these constants to (10),

$$
x=x_{0} \cos \omega t+\frac{v_{x}^{0}}{\omega} \sin \omega t
$$

or

$$
x=x_{0} \cos \omega t+\frac{v}{\omega} \sin \varphi_{0} \sin \omega t
$$

because the initial velocity $v_{x}^{0}$ (horizontal projection of peripheral velocity $v$ of weight in the assumed initial point)

$$
v_{x}^{0}=v \sin \varphi_{0}
$$

The symbol $v_{x}^{0}$ cannot be interpreted as $v_{x}$ raised to zero power. That later option corresponds with the record $\left(v_{x}\right)^{0}$, and this simply equals one. The notations, as can be noticed, have also important interpretation meaning. Regarding further that $x_{0}=A \cos \varphi_{0}$ (see Fig. 3), and $v: \omega=A$, that results from a known relationship between the peripheral and angular velocities, one obtains

$$
x=A \cos \varphi_{0} \cos \omega t+A \sin \varphi_{0} \sin \omega t
$$

or

$$
x=A \cos \left(\varphi_{0}-\omega t\right)
$$

It is worth explaining now where the equation (1) comes from, which is commonly used and generally accepted on the cognitive ground of oscillation reality. For that it is enough to derive the first and then the second derivatives of function (1), and then that first dependence and its derivative of the second order in the fictional-real force equilibrium, recorded as follows:

$$
\Delta B_{x}=\Delta S_{x}
$$


that was earlier mentioned in the text.

Therefore

$$
\begin{gathered}
\dot{x}=\frac{d x}{d t}=-A \omega \sin \left(\varphi_{0}+\omega t\right) \\
\ddot{x}=\frac{d^{2} x}{d t^{2}}=-A \omega^{2} \cos \left(\varphi_{0}+\omega t\right)
\end{gathered}
$$

and then

$$
\begin{gathered}
\Delta B_{x}=-m \ddot{x}=m A \omega^{2} \cos \left(\varphi_{0}+\omega t\right) \\
\Delta S_{x}=k x=k A \cos \left(\varphi_{0}+\omega t\right)
\end{gathered}
$$

with the dependence (27) obtained after taking into account the known definition of inertia force, and (28) after complying with the record (8) and the definition (also known) of the coefficient of stiffness.

Substituting (27) and (28) to (24) one obtains finally

$$
-m \ddot{x}=k x
$$

or

$$
m \ddot{x}+k x=0
$$

which is the earlier recorded formula (1).

Complying the extended records (27), and (28) with the formula (24) gives the following results:

$$
m A \omega^{2} \cos \left(\varphi_{0}+\omega t\right)=k A \cos \left(\varphi_{0}+\omega t\right)
$$

so

$$
\omega=\sqrt{\frac{k}{m}}
$$

which is that was determined by formula (6) and named the frequency of free vibration of the considered system.

In all these considerations, according to the existent knowledge, it may be noticed that mathematics really veiled/concealed the essence of the problem. The physical aspect is then in the shadow of cognition. In further part of this work that question will be explained and considered concerning the adequacy of record.

\section{CRITICAL ANALYSIS OF EXISTENT RECORD ON VIBRATIONS OF THE VERTICAL MASS-ELASTIC SYSTEM}

Analysis of the existent record of vibrations of the vertical mass-elastic system must be surely critical because the whole this record is inadequate and does not respond to the studied phenomenon of vibrating motion. The critics of description of this phenomenon will be 
carried out on three different planes: notion, physical, and mathematical. All they will have philosophical references.

First it should be noted that the path/way length of weight should be analyzed instead of its position determined by a coodinate. The path/way length, rising continuously which results of its nature, is a physical magnitude of which consecutive derivatives are determined magnitudes, also physical. Determination of derivatives of the weight coordinates has no sense, as de facto it does not lead to obtain nor a velocity nor an acceleration. They are only some mathematical forms assuming the values: negative, zero, and positive. It is known, however, that the physical magnitudes are positive only and may be measured.

The assumed fictional-real model of the mass-elastic system of which the force equilibrium/balance is brought about to the equality of the elastic force increment and decrease of inertia force; it leads to erroneous conclusion that the so called frequency of free vibration (in fact it concerns the angular velocity, here just apparent) does not depend on the amplitude (extensity of vibrations) and only on the weight mass and stiffness of elastic element. It may be recorded as follows:

$$
\begin{aligned}
& m\left(\omega_{0}\right)^{2} A_{0}=k A_{0} \\
& m\left(\omega_{1}\right)^{2} A_{1}=k A_{1} \\
& m\left(\omega_{2}\right)^{2} A_{2}=k A_{2} \\
& \cdots \cdots \cdots \cdots \cdots \cdots \cdots \cdots \\
& m\left(\omega_{i}\right)^{2} A_{i}=k A_{i}
\end{aligned}
$$

from which it results that

$$
\omega_{0}=\omega_{1}=\omega_{2}=\ldots=\omega_{i}=\sqrt{\frac{k}{m}}
$$

In fact that magnitude is simply the relative coefficient of stiffness $k_{m}$, that is the coefficient referred to the mass $m$ of weight. Thus

$$
k_{m}=\sqrt{\frac{k}{m}}
$$

If one has to interpret the displacement of shadow/projection of rotating weight as the simple harmonic motion, so let the equilibrium of rotating mass-elastic system be recorded adequately. The condition for the force equilibrium is presented as follows:

$$
B+S=F
$$

where: $B$ - the inertia force, real inertia force, $S$ - elasticity force, $F$ - centrifugal force. The graphic formulation of this equilibrium (Fig. 4) indicates that all these forces have radial direction and their projections on the direction of the oscillator motion correspond with this apparent harmonic motion, that is

$$
B_{x}+S_{x}=F_{x}
$$


(a))
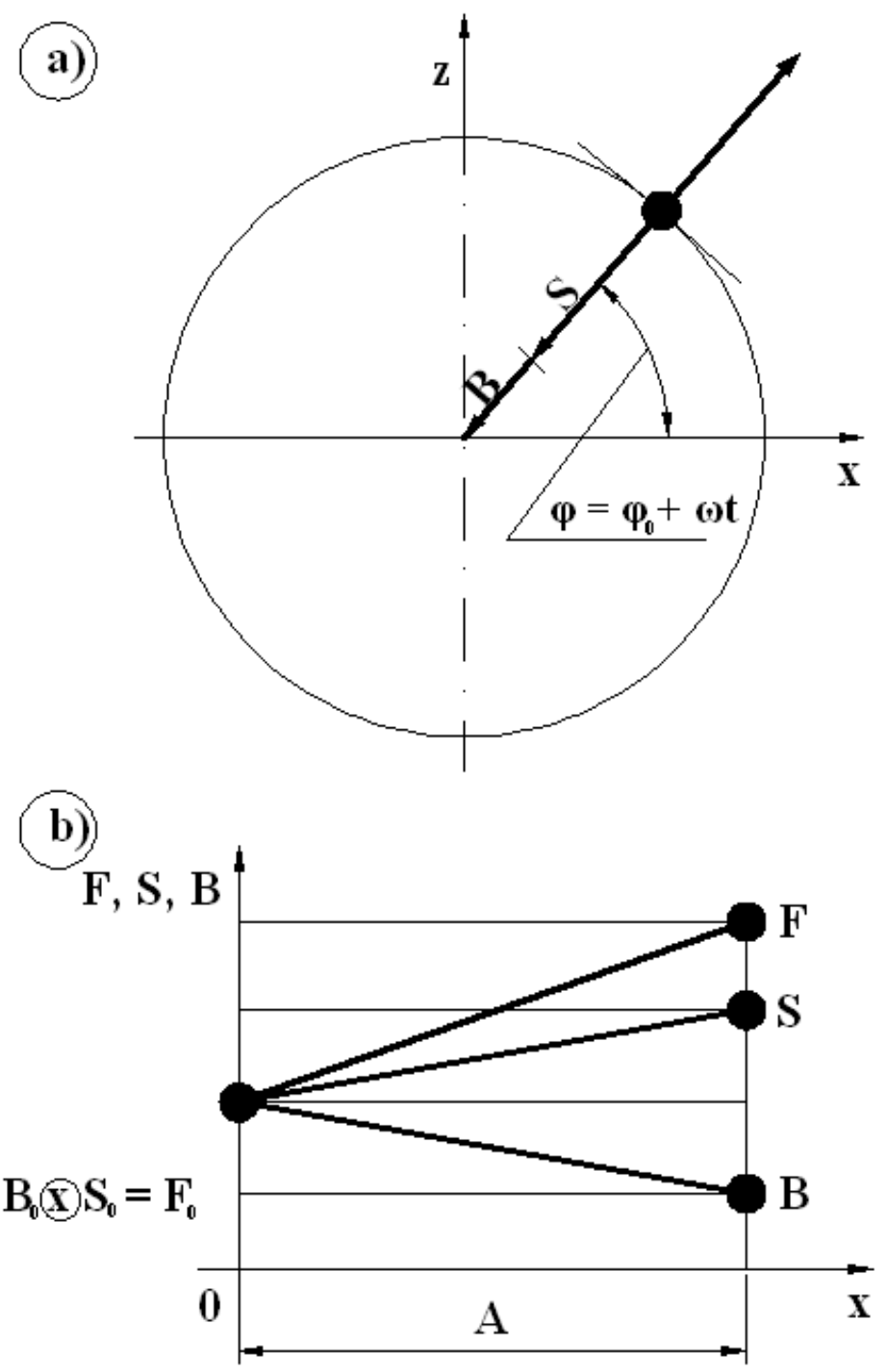

Fig. 4. Real force characteristics of rotating mass-elastic system with the motion of weight projection having oscillation character.

All those forces on the direction of oscillator motion may be recorded as follows:

$$
\begin{gathered}
B=B_{0}-b x \\
S=S_{0}+k x \\
F=F_{0}+m \omega^{2} x
\end{gathered}
$$

where the symbols $S_{0}, B_{0}, F_{0}$ denote initial forces of inertia, elasticity, and centrifugal, respectively, with the values of these forces being equal, and the equality of these forces; inertia, and elasticity results from their co-existence, corresponding with energetic character 
of the system under its stable state. The described characteristics of the system is presented by the symbols $B_{0} \otimes S_{0}=F_{0}$. This characteristics is characterized by the graphical approach (Fig. 4b).

The unstable equilibrium, expressed by the record (36), after substituting the formulae (37), (38), and (39) to it, takes the following form:

$$
B_{0}-b x+S_{0}+k x=F_{0}+m \omega^{2} x
$$

After taking into account that $B_{0} \otimes S_{0}=F_{0}$, the formula may be recorded as

$$
m \omega^{2} x+b x+k x=B_{0}
$$

and further, knowing that

$$
B_{0}=m a_{0}
$$

one obtains the following form of equation (41)

that

$$
m \omega^{2} x+b x+k x=m a_{0}
$$

$$
\omega^{2}=\frac{a_{0}}{A}-\frac{b}{m}-\frac{k}{m}
$$

where: $\omega$ - real angular velocity of the system, $a_{0}$ - initial acceleration of weight, $b-$ directional coefficient of inertia force (coefficient of inertia), $k$ - directional coefficient of elasticity force (coefficient of stiffness, here the elastic stiffness, or elasticity).

As can be seen, that real angular velocity (not an apparent one, called sometimes circular frequency, angular frequency, and even the frequency of free vibration) depends not only on the constant $k$ and mass $m$, but also many other system parameters, separated and explained above.

However, there is still lack of adequate description of harmonic vibrations of weight, the vibrations in one plane, the vibrations not connected with any rotating motion. This gap will be fulfilled with further considerations. They are to lead, by reasoning, to the adequate record of harmonic motion of vertically situated elastic oscillator.

\section{ENERGETIC STATES OF OSCILLATOR}

The adequate description of the free harmonic motion of oscillator, being the subject of this work, cannot be based on existent erroneous interpretation of its motion. The existent knowledge contains some elements having features of artifacts. They seem to be artificial, schematic, dead, just deforming the reality. The above presented critical analysis of the subject serves to explain the shortcoming of the reasoning.

A proper adequate description of the phenomenon has to relate to the real free variable motion of the oscillator taking place in one plane, without any references to a projection, shadow, and other characteristics of this type imitating harmonic motion. The initial conditions should relate to the beginning of the considered phenomenon and not to any 
assumed moments of measurements of determined magnitudes. No voluntary approaches tending to apparent equilibrium of forces are acceptable. The variable motion taking place in the space-time is characteristic with no equilibrium, also the force one.

Further considerations are to develop the subject providing with some cognitive quests as well. Therefore a proper energetic foundation to describe the harmonic motion phenomenon is very important. The energetic states of the vibrating system, being the states on the ends of the space-times or potential fields, where the instantaneous or energetic equilibrium exists, should be taken into account. This is the only equilibrium which takes place in a free motion. However, in the space-time there is no (also energetic) equilibrium.

The considered mass-elastic system is at first in a stable static energetic state (Fig. 5a). It possesses the following state, as being on a stable static potential field (SSPF), with the equality of the force co-existence system

$$
B_{0} \otimes S_{0}=B_{0} \otimes S_{0}
$$

where the symbol $\otimes$ denotes the state of co-existence of forces of inertia $B_{0}$ and elasticity $S_{0}$.

From the energetic viewpoint it is the most proper force presentation. That description allows for fulfilling the rule of energy conservation.

Now the action of external stimulus appears (Fig. 5b), which acts on the system first with the force $F_{0}$. The equality of the force co-existence comes round of inertia $B_{0}$ and elasticity $S_{0}$, expressed by the following record:

$$
B_{0} \otimes S_{0}=F_{0}
$$

A stable static energetic state has been distorted that means the beginning of system transition to the neighbouring potential field. That is unstable static potential field (ASPF). Such a transition progresses through a space-time (dotted area in Fig. 5). There is no force equilibrium. All forces change their values: the active (driving) force $F$ rises, the elastic (passive) force $S$ also rises, whereas the inertia force (passive) drops, respectively. The courses are linear as they are expressed as a function of way $x$.

On the unstable static potential field (Fig. 5c) the equality of forces occurs, that is

$$
B_{1}+S_{1}=F_{1}
$$

and this corresponds also to the energetic equilibrium, unstable static energetic equilibrium.

Here the rule of energy conservation is fulfilled, which in a quantitative approach corresponds with the equality of measure sums of all energies or their potentials. One may record it as follows:

$$
2 V_{B}^{(0)}+2 V_{S}^{(0)}=V_{B}^{(1)}+V_{S}^{(1)}+V_{F}^{(1)}
$$

where: $V_{B}^{(0)}$ - inertia potential on the initial zero potential field; $V_{S}^{(0)}$ - elastic potential on this field; $V_{B}^{(1)}$ - inertia potential on the neighbouring first potential field; $V_{S}^{(1)}$ - elastic potential on this field; $V_{F}^{(1)}-$ driving potential on this field. 


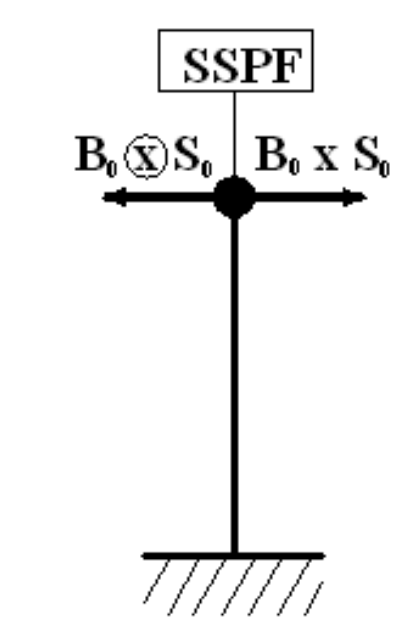

(a)

$B_{0}(\mathbf{s}) S_{0}=B_{0}(\mathbf{x}) S_{0}$
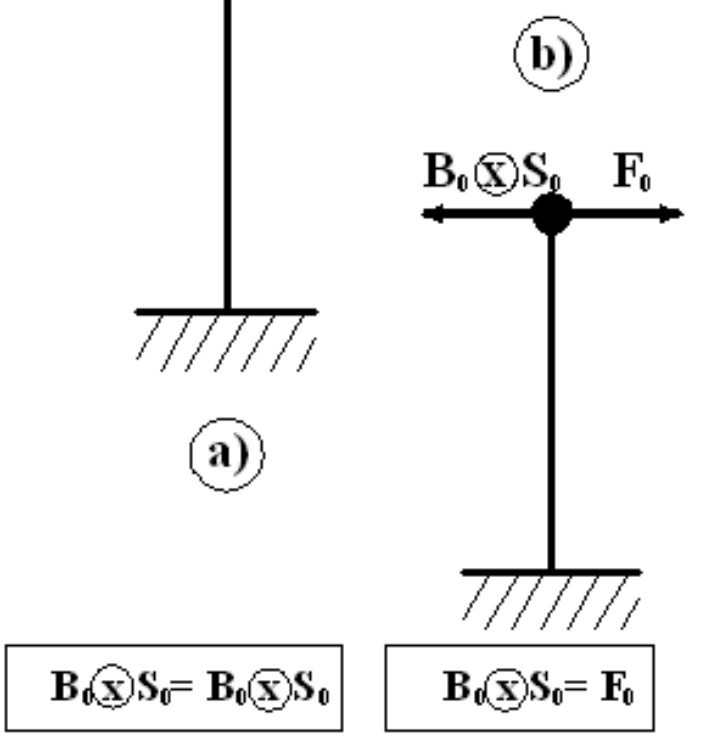

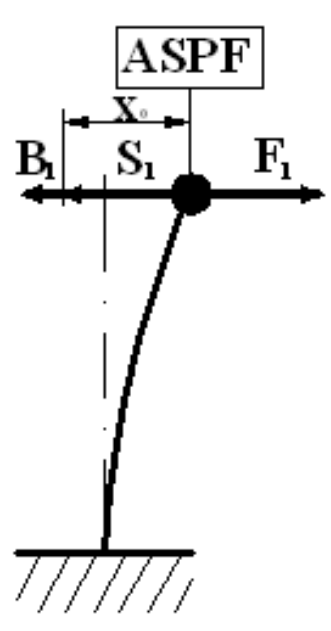

(c)
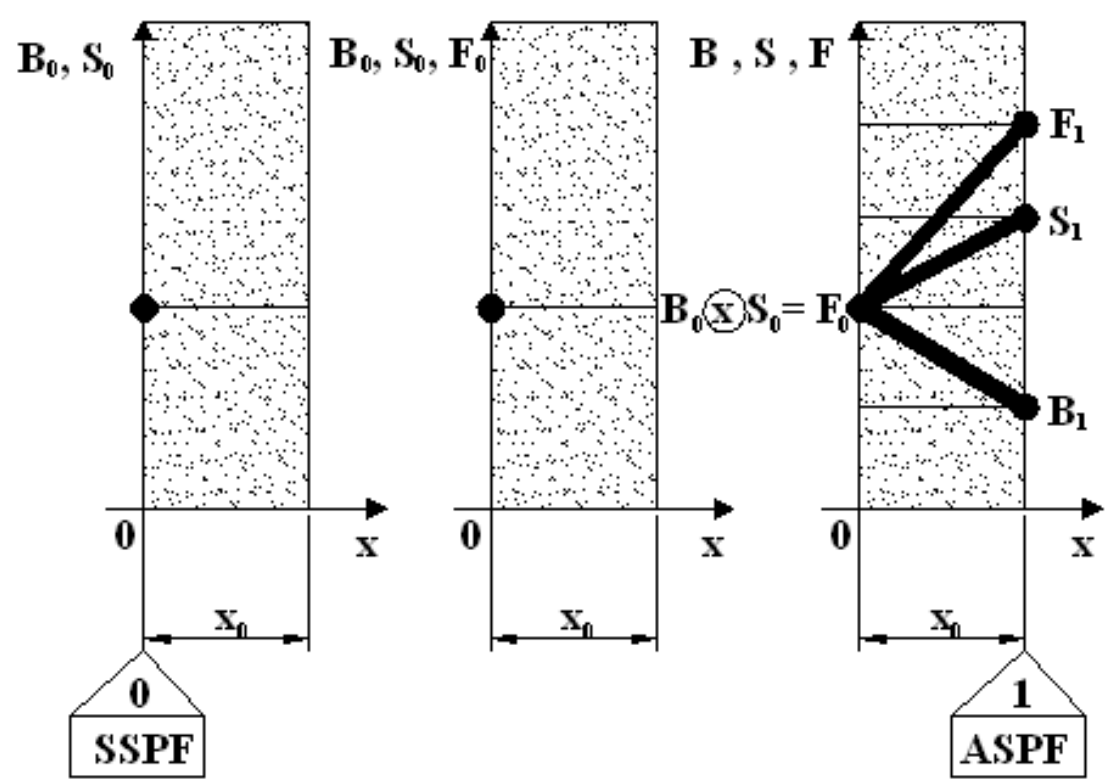

Fig. 5. Scheme of transition of mass-elastic system form the stable static potential field (SSPF) to the unstable field of this type (ASPF).

Regarding the potential is equal to the product of field intensity (which is the force on the field) and the length of space-time (here this characteristics is denoted by a symbol $x_{0}$ ), one may precise the record (48), so that

$$
2 B_{0} \cdot x_{0}+2 S_{0} \cdot x_{0}=B_{1} \cdot x_{0}+S_{1} \cdot x_{0}+F_{1} \cdot x_{0}
$$


From this a determined dependence between forces (intensities of potential fields) is as follows,

$$
2 B_{0}+2 S_{0}=B_{1}+S_{1}+F_{1}
$$

and regarding (47) also that

$$
B_{0}+S_{0}=F_{1}
$$

That was a forced motion needed to transfer the system on the neighbouring potential field where the analysed herewith the phenomenon of harmonic motion begins. After stopping the interference of external stimulus the mass-elastic system begins its free motion being in character the simple harmonic motion. It will cross the space-times with a variable motion with accelerated variable motion and this kind of retarded motion. Of course, in a determined space-time there is only one kind of variable motion.

\section{FORCE CHARACTERISTICS OF FREE HARMONIC MOTION OF A VERTICAL OSCILLATOR}

One could simulate a forced vibrating motion taking into account an extended fictionreal force equilibrium and particular changes. However, such an approach has no justification. A description of real force characteristics of the vertical oscillator motion, especially free of that motion kind is necessary.

It should be noted that there is no real force equilibrium when crossing one system to another. That is valid both in forced and free motion. Thus the courses of each separate force should be described. The up-to-date approach, relying on creating fiction under the d'Alembert's rule to form an apparent equilibrium, is completely unjustified. It would be in contradiction to search for a natural reality.

There is an energetic equilibrium only the essence of which has been clarified above in a previous section. Such an equilibrium takes place in a forced motion and in free harmonic motion. In the last case the force equilibrium appears not on all potential fields. It is on the stable potential fields but not on the unstable fields of this kind. That is to be clarified furthermore.

The action of external stimulus with the force $F_{l}$ results in getting the system from a stable state into unstable one (Fig. 6). That means the oscillator moved from a stable static potential field (SSPF) onto an unstable one (ASPF). When the stimulus stops acting, a free harmonic oscillator motion begins. That means the accelerated motion appears first, and then a retarded one. An increase in the inertia force $B$, and decrease of elastic force $S$ correspond with the accelerated motion, whereas the decrease in inertia force and increase in elastic force correspond with the retarded variable one. Furthermore these force variations are repeated cyclically.

It is worth noting that all these courses are linear. They may change their nature in case the time of system motion has been regarded. The force curves (Fig. 7) will have an exponential character, progressively and degressively rising or decreasing, respectively. 


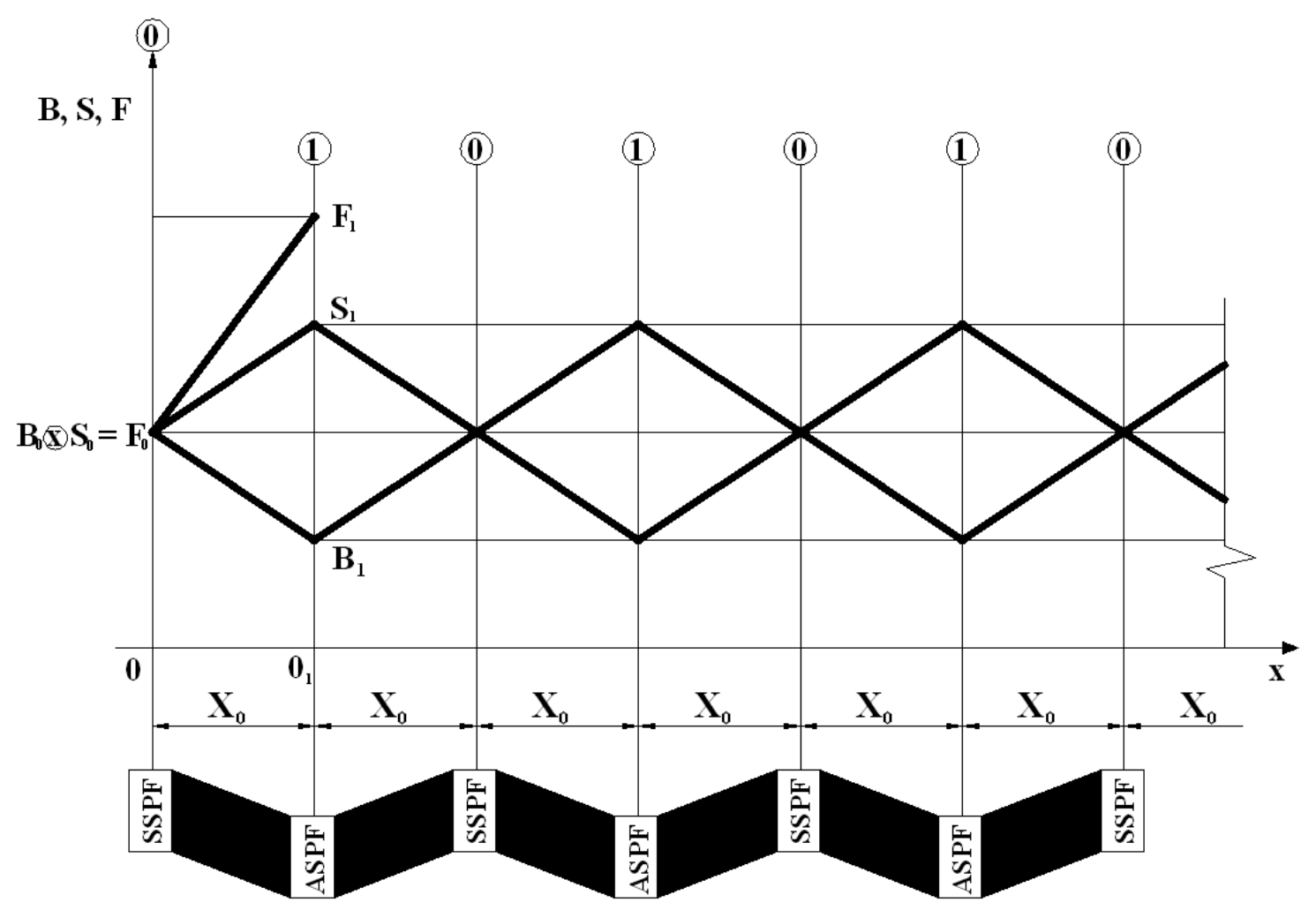

Fig. 6. Force-path/way characteristics of free harmonic vertical motion of oscillator.

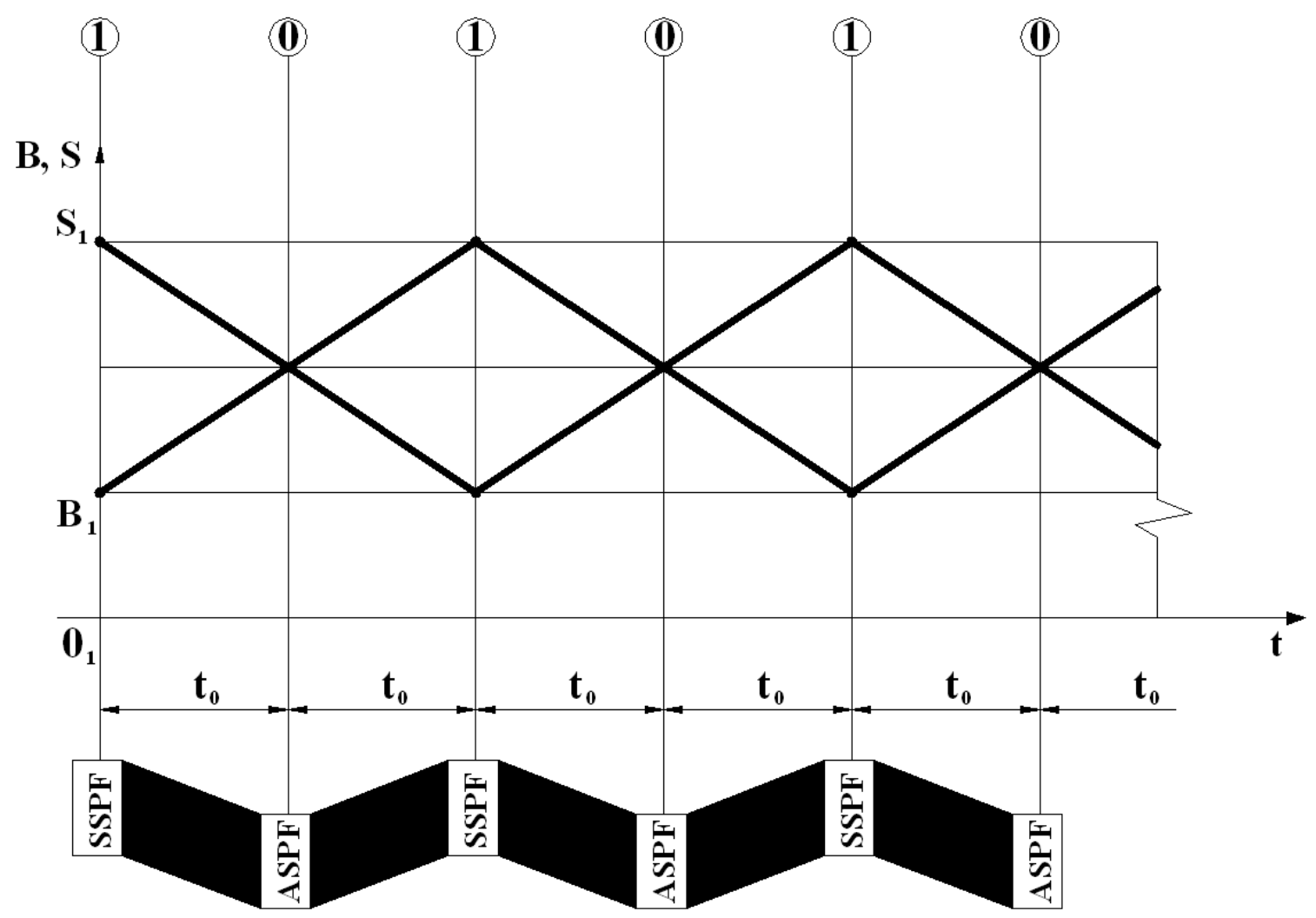

Fig. 7. Force-time characteristics of free harmonic vertical motion of oscillator. 
The courses of the forces, both path/way and time, may be described, knowing that

$$
\begin{aligned}
& B=m a \\
& S=k x
\end{aligned}
$$

where the symbol $a$ denotes a real acceleration, and that means the force $B$ is that real (not fictional!) inertia force. The contents of other symbols are known. One should admit, however, that $x$ is not a coordinate of oscillator position anymore, but the length of its way in a determined space-time, i.e. between the neighbouring potential fields. However, first a source equation of the way length of the system should be presented. The equation will allow for determination further, derivative dynamic (kinetic and force) characteristics.

\section{GENERAL SOURCE EQUATION OF THE PATH LENGTH AND RESULTING DETAILED FORMS}

The source differential equation describing a variety of phenomena and processes [1121], proceeding with non-linearly variable intensity, has the following form:

$$
d Z= \pm \frac{\partial Z}{\partial N} d N
$$

where: $d Z$ - total differential of dependent variable, $d N$ - total differential of independent variable, $\partial Z / \partial N$ - partial derivative of dependent variable referred to independent variable. The signs $( \pm)$ are the algebraic operators fulfilling a determined role. The sign $(+)$ has a formal meaning, confirming a physical essence of determined relationship. The sign (-) provides such a meaning to a determined record.

For the considered variables the formula (54) has the following form:

$$
d x= \pm \frac{\partial x}{\partial t} d t
$$

because the way length $x$ is a dependent variable, and the time of oscillator motion $t$ is an independent variable. The way of detailed solution of this type of equation has been presented earlier $[11,12]$. Here the solutions relating to variable accelerated motion and the following retarded motion will be given. That corresponds with the half-cycle of oscillator motion. The dependence $x(t)$ for the first motion is exponential and rising progressively (Fig. 8), whereas the second motion possesses degressively rising exponential character.

Here is the dependence of the path/way length $x$ on time $t$ for the accelerated motion

$$
x=x_{0}\left(e^{\frac{t}{\Theta}}-1\right)
$$

where $x_{o}$ denotes the length of space-time being between an unstable static potential field (ASPF) and a stable field of this kind, i.e. SSPF. 


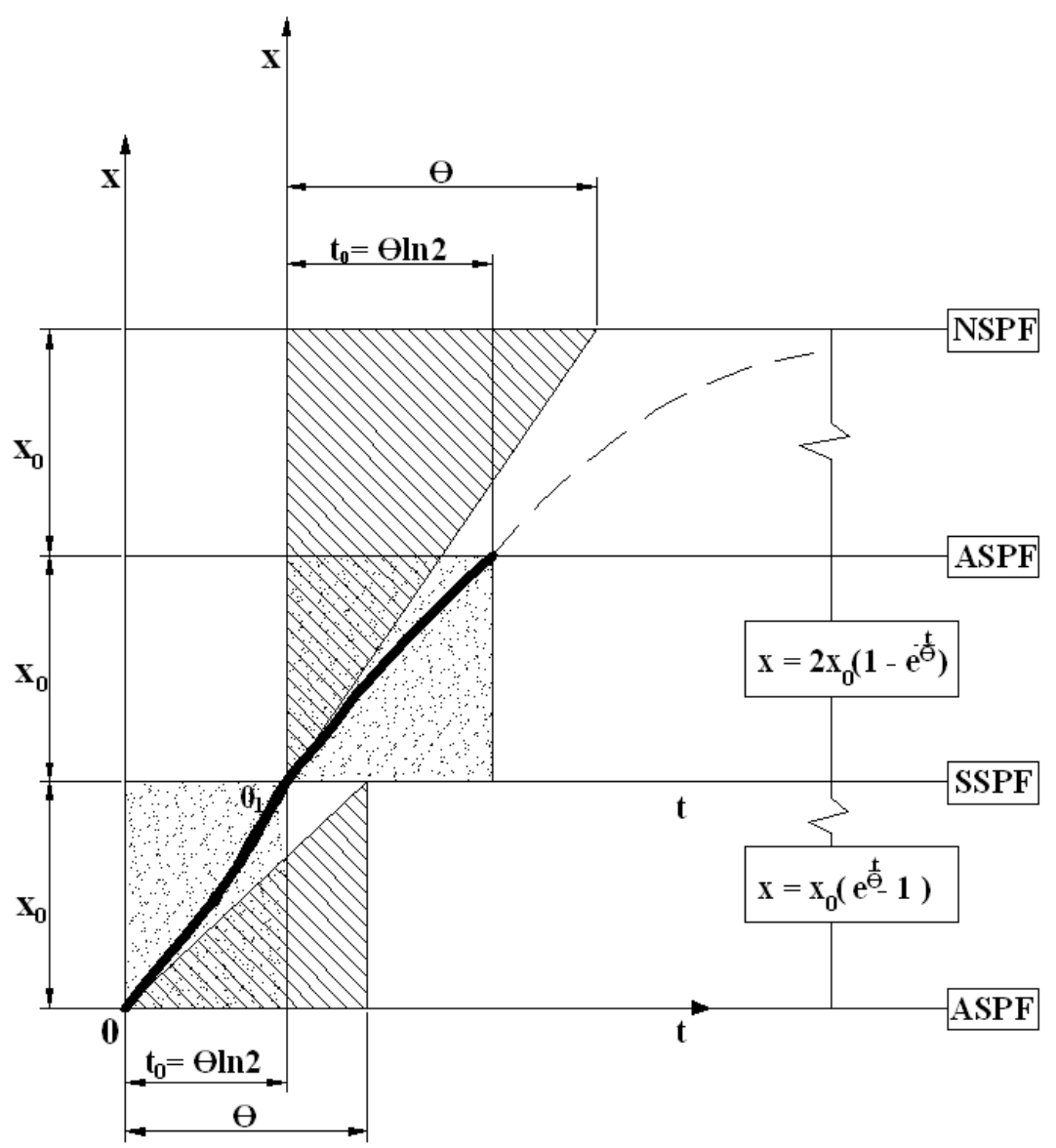

Fig. 8. Indicative dependence of the path/way length on time for two neighbouring space-times.

The symbol $\Theta$ corresponds to the so called time constant, being the time of transition of a body onto the neighbouring potential field with an initial invariable velocity. That velocity corresponds with the tangent of angle to the curve in the initial point 0 .

The length $x_{0}$ of the space-time is the coordinate of a terminal point $0_{1}$ of this course with the point occurring on a neighbouring potential field. The second coordinate, abscissa, being the time $t_{0}$ of accelerated motion may be determined by substituting $x=x_{0}$ to (56). That leads to the result

$$
t_{0}=\Theta \ln 2
$$

The dependence $x(t)$ for a retarded motion, taking place in the next real space-time, takes the following form:

$$
x=2 x_{0}\left(1-e^{-\frac{t}{\Theta}}\right)
$$

Here the time constant $\Theta$ is at the height of the nominal static potential field (NSPF), that corresponds with an asymptote to which the variable $x$ is approaching. It is visible the real course of the function ends at the unstable static potential field (ASPF), then the curve 
(dashed line in Fig. 8) permeates the apparent space-time being between the potential fields mentioned above. That auxiliary design made it possible to separate physical part of the described reality by isolating it from its apparent (fictional) fragment.

Naturally, one may further develop description of the considered phenomenon of harmonic motion, creating consecutive time characteristics by providing further derivatives of the first, second, third order, etc. These characteristics will form the oscillator kinetics. Considering particular forces one obtains the description of its dynamics.

Following the procedure one may determine particular works, momentums, timeeffects, dynamic moments, and even energy measures which are potentials, i.e. a variety of characteristics may be obtained, being both functional and parametric. The adequate, source characteristics (56), (58) allow to develop the set of all these characteristics, as it is only on one-side closed, just at the side of the source, that is the primary description of the studied reality.

\section{CONNECTIONS BETWEEN THE CHARACTERISTICS OF MOTION, REAL AND SIMULATED}

Now one may determine in a right way the so called angular frequency/pulsation resulting from the equation (5) and simulating one-way oscillator motion. This magnitude is obtained by comparison of real vibration period $T$ of oscillator with the fictional vibration period $T_{0}$ of the apparent rotary motion.

The real vibration period $T$ is the time of double motion cycle of mass-elastic system, that is the time of its transition through four consecutive space-times, then

$$
T=4 t_{0}
$$

and after taking into account (57)

$$
T=4 \Theta \ln 2
$$

It results from equation (5) that

$$
T_{0}=\frac{2 \pi}{\omega}
$$

Therefore, comparing the formulae (60) and (61), one obtains

$$
4 \Theta \ln 2=\frac{2 \pi}{\omega}
$$

and

$$
\omega=\frac{\pi}{2 \Theta \ln 2}
$$

Next, one may prove that the magnitude $T_{0}$ depends also on the initial deflection, and to say more closely - on the amplitude, a determined vibration amplitude, that is $x_{0}$. It also depends on the mass $m$ of weight and the proper stiffness of elastic element, that is the initial force of elasticity $S_{0}$. 
To do this, the equality of forces in the free oscillator motion should be taken into account which takes place on a stable static potential field (see Figs. 6 and 7). It concerns the forces of inertia $B_{0}$ and elasticity $S_{0}$.

To obtain the form of formula on the inertia force, firstly one has to determine the acceleration $a_{0}$ on the stable static potential field. It is two times higher than the initial acceleration $a_{1}$, that results from the time coordinates $t=0$ and $t_{0}=\Theta \ln 2$, which are substituted to the dependence $a=f(t)$. This dependence, in turn, is the second derivative of function (56), then

$$
a=\frac{d^{2} x}{d t^{2}}=\frac{x_{0}}{\Theta} e^{\frac{t}{\Theta}}=a_{0} e^{\frac{t}{\Theta}}
$$

For $t=0, a=a_{0}=x_{0}: \Theta^{2}$, and for $t_{0}=\Theta \ln 2$ it is really $a_{1}=2 a_{0}$.

The force $B_{0}$ will be equal

$$
B_{0}=m a_{1}=2 m a_{0}=\frac{2 m x_{0}}{\Theta^{2}}
$$

and after considering the equality $S_{0}=B_{0}$

$$
S_{0}=\frac{2 m x_{0}}{\Theta^{2}}
$$

From the equation (66)

$$
\Theta=\sqrt{\frac{2 m x_{0}}{S_{0}}}
$$

that after substituting to (63), and (61), gives

$$
T_{0}=\frac{2 \pi}{\omega}=4 \Theta \ln 2=4 \ln 2 \sqrt{\frac{2 m x_{0}}{S_{0}}}
$$

It appears the magnitude $T_{0}$ depends on mass $m$, the proper elasticity force $S_{0}$ and the amplitude of vibration $x_{0}$. Therefore, the up-to-date assumption that $T_{0}$ does not depend on the deflection of oscillator weight is erroneous and cannot be referred to on the ground of adequate knowledge presented.

\section{CONCLUSION}

In summary it is worth underlining that the discovered differences between existent and a new adequate description of oscillating vertical motion of mass-elastic system are fundamental and meaningful. A classical academic approach to this problem only simulates the studied reality. Harmonic oscillating one-way motion, cyclically variable in fact, has been 
substituted by a fictional rotary motion. The coordinates of this variable motion form an apparent picture of vibrating motion.

It would be reasonable to introduce this adequate knowledge on the ground of science and pass it for practical applications. The proposed theory is closely connected with the reality and its adequacy means full applicability to practice.

\section{References}

[1] J. R. Taylor, Classical mechanics (in Polish), Vol. 1 and 2 (transl. from English), $1^{\text {st }}$ ed. by PWN SA, Warszawa 2006.

[2] S. Ziemba, Vibration analysis (in Polish), ed. by PWN, Warszawa 1957.

[3] K. Piszczek, J. Walczak, Vibrations in machine building (in Polish), 2nd ed. by PWN, Warszawa-Kraków 1967.

[4] Z. Osiński, Theory of vibrations (in Polish), ed. by PWN, Warszawa 1978.

[5] Dąbrowski Z., Przegląd Mechaniczny 11 (2007) 15-18.

[6] A. Okniński, B. Radziszewski, Przegląd Mechaniczny 1 (2009) 32-36.

[7] M. di Bernardo, C. J. Budd, A. R. Champneys, P. Kowalczyk, Piecewise-Smooth Dynamical Systems. Theory and Applications, Springer-Verlag, Berlin 2008.

[8] M. Mita, M. Arai, S. Tensaka, D. Kobayashi, H. Fujita, IEEE Journal of Microelectromechanical Systems. 12(1) (2003) 37-41.

[9] A. Okniński, B. Radziszewski, Stability and bifurcations in certain systems with impacts, Proc. 8th Conference on Dynamical Systems Theory and Application, Vol. 1, 2003, pp. 387-394.

[10] A. Grządziela, Przegląd Mechaniczny 4 (2009) 17-20.

[11] Zdzisław Pluta, Tadeusz Hryniewicz, Intern. J. Adv. Mater. Technol. 62(5) (2012) 529-542, DOI: 10.1007/s00170-011-3813-5.

[12] Zdzisław Pluta, Tadeusz Hryniewicz, Journal of Modern Physics 3(7) (2012) 625-635; DOI: $10.4236 / j m p .2012 .37086$.

[13] Zdzisław Pluta, Tadeusz Hryniewicz, International Letters of Chemistry, Physics and Astronomy 2 (2012) 28-34.

[14] Zdzisław Pluta, Tadeusz Hryniewicz, International Letters of Chemistry, Physics and Astronomy 3 (2012) 1-10.

[15] Zdzisław Pluta, Tadeusz Hryniewicz, International Letters of Chemistry, Physics and Astronomy 3 (2012) 11-23.

[16] Zdzisław Pluta, Tadeusz Hryniewicz, International Letters of Chemistry, Physics and Astronomy 4 (2012) 1-7.

[17] Zdzisław Pluta, Tadeusz Hryniewicz, International Letters of Chemistry, Physics and Astronomy 4 (2012) 8-16.

[18] Zdzisław Pluta, Tadeusz Hryniewicz, International Letters of Chemistry, Physics and Astronomy 5 (2012) 35-45. 
[19] Zdzisław Pluta, Tadeusz Hryniewicz, International Letters of Chemistry, Physics and Astronomy 3 (2013) 67-84.

[20] Zdzisław Pluta, Tadeusz Hryniewicz, International Letters of Chemistry, Physics and Astronomy 3 (2013) 85-100.

[21] Zdzisław Pluta, Tadeusz Hryniewicz, International Letters of Chemistry, Physics and Astronomy 4 (2013) 56-72. 